\title{
TAGUNG
}

\section{Der Einfluss der öffentlichen Verwaltung auf die ökonomische Performance von Ländern}

\author{
Zareh Asatryan, Benjamin Bittschi, Melissa Engel, Lisa Evers, Friedrich Heine- \\ mann, Christoph Schröder, Frank Streif und Mustafa Yeter*
}

Nicht zuletzt vor dem Hintergrund der gegenwärtigen Krise hat die Rolle der öffentlichen Verwaltung im politischen Diskurs an Bedeutung gewonnen. So nimmt diese unter anderem eine prominente Position in der Wachstumsstrategie der Europäischen Union (Europe 2020) ein. ${ }^{1}$ Deshalb war es das Ziel der diesjährigen ,ZEW Public Finance Conference", die Bedeutung der öffentlichen Verwaltung für die ökonomische Performance auf unterschiedlichen Ebenen zu diskutieren.

\section{Hürden für eine fiskalische Konsolidierung}

Stefan Lamp untersuchte den Zusammenhang zwischen Steuerreformen und privaten Investitionen. Die von ihm vorgestellte Studie unterschied dabei zwischen Anpassungen der geplanten und tatsächlich realisierten Investitionsausgaben von Unternehmen in Reaktion auf exogene Änderungen in der Steuergesetzgebung. Die Ergebnisse zeigten, dass sowohl die geplanten Investitionsausgaben als auch die realisierten Investitionen in Reaktion auf Steuererhöhungen nach unten angepasst werden. Dieser Effekt sei für die geplanten leicht stärker als für die tatsächlichen Ausgaben. Darüber hinaus zeige sich, dass Unternehmen nicht nur auf verabschiedete Gesetzesänderungen reagierten, sondern auch auf diskutierte Reformvorschläge. Sie würden so ver-

\section{ZEW Public Finance Conference. Öffentliche Verwaltung und ökonomische Performance}

Zentrum für Europäische Wirtschaftsforschung (ZEW)

Mit finanzieller Unterstützung der Europäischen Kommission.

28.-29. April 2014, Mannheim

\section{Fiscal Consolidation}

Chair: Christoph SCHRÖDER, Zentrum für Europäische Wirtschaftsforschung, Mannheim

The Investment Effect of Fiscal Consolidation Stefan LAMP and Silvia ALBRIZIO, Organisation for Economic Co-operation and Development and European University Institute, Fiesole

Discussant: Ludovít ÓDOR

The Good, the Bad and the Ugly

Ludovít ÓDOR, Council for Budget Responsibility Slovakia, Bratislava

Discussant: Florian NEUMEIER

Public Attitudes Toward Fiscal Consolidation: Evidence from a Representative German Population Survey

Florian NEUMEIER and Bernd HAYO, PhilippsUniversität Marburg

Discussant: Christoph SCHRÖDER

\footnotetext{
* Alle Autoren sind Mitarbeiter/innen des Zentrums für Europäische Wirtschaftsforschung, Abteilung Unternehmensbesteuerung und Öffentliche Finanzwirtschaft, Mannheim.

1 Europäische Kommission: Mitteilung der Kommission. Europa 2020. Eine Strategie für intelligentes, nachhaltiges und integratives Wachstum, KOM (2010) 2020.
} 
suchen, Konsequenzen zukünftiger Steuerrechtsänderungen vorwegzunehmen.

Ludovit Ódor evaluierte die Fiskalregeln des neuen europäischen fiskalpolitischen Regelwerks (6-Pack, 2-Pack, Europäischer Fiskalpakt) auf Basis der Kriterien Transparenz, Flexibilität, Durchsetzbarkeit, Zweckdienlichkeit, Effizienz, Simplizität und Widerspruchsfreiheit. Als problematisch erweise sich unter anderem die Fülle verschiedener und teilweise sogar widersprüchlicher Fiskalregeln, die zu einer Überregulierung führe. Zudem würden oft numerische Vorgaben gemacht, die in der Praxis schwierig zu evaluieren seien. So basiere die Berechnung des strukturellen Defizits beispielsweise auf Echtzeitschätzungen der Produktionslücke, für deren Berechnung es aber keine einheitlichen Richtlinien gebe. Ódor schlug daher eine generelle Vereinfachung und Vereinheitlichung der Regeln vor.

Florian Neumeier präsentierte eine Studie zu den Präferenzen der deutschen Bevölkerung in Bezug auf potenzielle Reformen zur fiskalischen Konsolidierung. Deren Autoren nutzten hierzu repräsentative Umfragedaten und versuchten, die Präferenzen der Bevölkerung im Hinblick auf die Dringlichkeit sowie die expliziten Maßnahmen der fiskalischen Konsolidierung mithilfe der persönlichen Merkmale der Umfrageteilnehmer zu erklären. Bezug nehmend auf die Präferenzen hinsichtlich der Dringlichkeit der Konsolidierung zeige sich, dass vor allem die eigene wirtschaftliche Situation, die persönlichen Zeitpräferenzen, die individuelle Informiertheit über die aktuelle fiskalische Situation des Bundes und das $\mathrm{Ma} \beta$ an Vertrauen, das die Umfrageteilnehmer Politikern entgegenbringen, einen maßgeblichen Einfluss auf die Präferenzbildung haben.

Christoph Schröder referierte zu den Bestimmungsgründen von seriellen (das heißt kurzfristig aufeinanderfolgenden) Umschuldungen souveräner Staaten im Zeitraum von 1970 bis 2010. Die Analyse konzentrierte
Haircut Size, Haircut Type and the Probability of Serial Sovereign Debt Restructurings

Christoph SCHRÖDER, Zentrum für Europäische Wirtschaftsforschung, Mannheim

Discussant: Stefan LAMP

\section{Public Administration I}

Chair: Mustafa YETER, Zentrum für Europäische Wirtschaftsforschung, Mannheim

Tax Compliance, Social Norms and Institutional Quality: An Evolutionary Theory of Public Good Provision

Panayiotis NICOLAIDES, European Commission, DG Taxation and Customs Union, Brussels

Discussant: Luca SALVADORI

Empirical Evidence on Tax Information Sharing among Sub-Central Administrations

Luca SALVADORI, José María DURÁNCABRÉ and Alejandro ESTELLER-MORÉ, Institut d'Economia de Barcelona (IEB) and Universitat de Barcelona

Discussant: Anders JENSEN

Information trails, tax enforcement and tax compliance

Anders JENSEN, London School of Economics, London; and Pierre BACHAS, University of California, Berkeley

Discussant: Panayiotis NICOLAIDES

Keynote I: Incentive Schemes for Local Government

Ben LOCKWOOD, University of Warwick

\section{Taxation \& Redistribution}

Chair: Benjamin BITTSCHI, Zentrum für Europäische Wirtschaftsforschung, Mannheim

Federal Income Tax Revenue Volatility Since 1966

Christopher BALDING, Peking University, Peking; and Estelle P. DAUCHY, New Economic School, Moscow

Discussant: Silvia AVRAM

The distributional effects of personal income tax expenditure

Silvia AVRAM, University of Essex

Discussant: Peter BACKUS 
sich dabei auf Höhe und Art der Schuldenschnitte. So könnten Staaten im Falle von drohenden oder realisierten Zahlungsrückständen mit ihren Gläubigern beispielsweise eine Verringerung des Nominalbetrags der fälligen Schulden, Laufzeitverlängerungen und/oder Zinsreduktionen aushandeln. Die Ergebnisse scheinen die Spekulationen des Internationalen Währungsfonds zu bestätigen, dass höhere Schuldenschnitte die Wahrscheinlichkeit weiterer Nachfolgeumschuldungen verringern könnten. Außerdem hätten Verringerungen des Kapitalwerts der neu verhandelten Kreditverträge, die durch eine Kürzung des Nominalbetrags zustande kamen, einen größeren negativen Einfluss auf die Wahrscheinlichkeit von Folgeumschuldungen als Kürzungen, die durch Laufzeitverlängerungen oder Zinsreduktionen herbeigeführt wurden. Dies scheine der theoretischen ökonomischen Logik zu widersprechen, die einen identischen Einfluss jedweder Verringerung des Kapitalwerts auf diese Wahrscheinlichkeit vorhersagen würde.

\section{Der Einfluss der Qualität der Steuerverwal- tung}

Panayiotis Nicolaides untersuchte in seinem Vortrag die theoretischen Determinanten für eine nutzenmaximierende Bereitstellung öffentlicher Güter in einem Modell mit dezentral agierenden Agenten, variierender institutioneller Qualität, der Möglichkeit der Steuerhinterziehung und unter Berücksichtigung sozialer Normen. Insbesondere untersuchte er die Interaktion zwischen diesen Faktoren. So zeige sich, dass im Falle einer effizienten Steuerverwaltung die Bedeutung sozialer Normen für eine Vermeidung von Steuerhinterziehung nachrangig sei. Nehme die Fähigkeit der effektiven Überwachung durch die Steuerverwaltung jedoch ab, wirkten die sozialen Normen wie deren Substitut. Vor diesem Hintergrund erlaube das Modell die Bestimmung des Umfangs sozial tolerierter Steuerhinterziehung.

Luca Salvadori präsentierte eine Studie zu den Anreizwirkungen für den Informations-
Other-regarding and self-insurance motives in demand for redistribution: an empirical analysis Peter BACKUS, University of Manchester and Institut d'Economica de Barcelona; and Alejandro ESTELLER-MORÉ, University of Barcelona and Institut d'Economia de Barcelona

Discussant: Benjamin BITTSCHI

On the Interaction of the Church Tax and Charitable Giving

Benjamin BITTSCHI, Sarah BORGLOH, Zentrum für Europäische Wirtschaftsforschung, Mannheim; and Berthold WIGGER, Karlsruhe Institut für Technologie, Karlsruhe

Discussant: Christopher BALDING

\section{B2-Fiscal Federalism \& Local Fiscal Policy}

CHAIR: Eckhard JANEBA, University of Mannheim

Analysis of Creative Accounting and its Impacts on Governments' Financial Performance: The Case of the Swiss Cantons

Nils SOGUEL and Maxime CLÉMENCEAU, Université de Lausanne

Discussant: Thushyanthan BASKARAN

Identifying local tax mimicking with administrative borders and a policy reform

Thushyanthan BASKARAN, Georg-August-Universität Göttingen

Discussant: Eckhard JANEBA

City Competition for the Creative Class

Eckhard JANEBA, University of Mannheim; and Thiess BÜTTNER, Friedrich-Alexander University Erlangen-Nuremberg

Discussant: Nils SOGUEL

\section{Tax Evasion}

Chair: Johannes RINCKE, Friedrich-AlexanderUniversity Erlangen-Nuremberg

Why do some firms evade taxes? Informality and the business environment in Uganda

Joseph MAWEJJE, Makerere University, Kampala

Discussant: Carolin HOLZMANN

Exploiting a Window of Opportunity: Multinationals' Profit Shifting in the Absence of Restrictions Carolin HOLZMANN, Friedrich-Alexander-University Erlangen-Nuremberg

Discussant: Tuomas KOSONEN 
austausch zwischen subnationalen Steuerverwaltungen in Spanien im Kontext der Vermögensteuer. Hierbei ergäbe sich Raum für ein strategisches Kalkül im Falle fehlerhafter Steuererklärungen und zu viel einbehaltener Steuern. So stünde die Steuerverwaltung vor der Abwägung, kooperativ zu sein, Informationsaustausch zu betreiben und die Steuereinnahmen umzuverteilen oder nicht kooperativ $\mathrm{zu}$ sein, keine Informationen weiterzugeben und zu viel entrichtete Steuern für sich zu behalten. Die empirische Analyse der Determinanten des Informationsaustausches zeige dabei, dass vorrangig die Reziprozität im Informationsaustausch der treibende Faktor für dessen Realisierung sei.

Anders Jensen untersuchte, ob größere und stärker von externem Kapital abhängige Unternehmen eine höhere Wahrscheinlichkeit für Betriebsprüfungen durch die Steuerverwaltung und bedingt hierdurch eine größere Steuermoral aufweisen. Hierzu verwendet die Studie Unternehmensdaten für mehr als 100.000 Firmen. Diese enthielten auch Informationen zur individuellen Steuermoral. Im Rahmen der empirischen Analyse zeige sich, dass die Unternehmensgröße sowohl eine wichtige Determinante für die Prüfungswahrscheinlichkeit als auch für die Steuermoral sei, dies jedoch nicht für die finanzielle $\mathrm{Ab}$ hängigkeit von externem Kapital gelte.

\section{Leistungsanreize für Politiker}

Ben Lockwood befasste sich in seinem Vortrag mit Leistungsanreizen für Politiker. Sein besonderes Augenmerk galt dabei den Folgen von quantifizierten Leistungsindikatoren. In England wurden in den Jahren von 2002 bis 2008 auf der kommunalen Ebene durch das sogenannte „Comprehensive Performance Assessment" solche Indikatoren eingeführt. Diese hätten in sechs zentralen Dienstleistungen die Effizienz der Kommunalpolitiker gemessen. Eine hohe Effizienz in der Leistungserbringung sei dabei durch einen größeren Spielraum bei der Entscheidungsfindung in ausgewählten Politikbereichen und durch
Do honest hairdressers get a haircut? On tax rate and tax evasion

Tuomas KOSONEN, Jarkko HARJU and Olli ROPPONEN, Government Institute for Economic Research, Helsinki

Discussant: Johannes RINCKE

Extrinsic vs Intrinsic Motivations for Tax Compliance: Evidence from a Field Experiment in Germany

Johannes RINCKE, Friedrich-Alexander-University Erlangen-Nuremberg; Nadja DWENGER, Max-Planck-Institut for Tax Law and Public Finance, Munich; Henrik KLEVEN, London School of Economics; and Imran RASUL, University College London

Discussant: Joseph MAWEJJE

\section{Fiscal Rules}

Chair: Friedrich HEINEMANN, Zentrum für Europäische Wirtschaftsforschung, Mannheim

The impact of fiscal rules on the stabilisation function of fiscal policy

Agnese SACCHI, Universitas Mercatorum, Rome; and Simone SALOTTI, Oxford Brookes University, Oxford

Discussant: Wildmer Daniel GREGORI

Fiscal Rules and Public Spending: Evidence from Italian Municipalities

Wildmer Daniel GREGORI, University of Bologna

Discussant: Heiko BURRET

Inter-Jurisdictional Effects of Fiscal Rules - The Swiss Experience

Heiko BURRET and Lars FELD, Walter Eucken Institute, Freiburg

Discussant: Wolf Heinrich REUTER

National Numerical Fiscal Rules: Not Complied With, But Still Effective?

Wolf Heinrich REUTER, Wirtschaftsuniversität Wien, Vienna

Discussant: Agnese SACCHI

\section{Incidence of Taxation}

Chair: Pierre BACHAS, University of California, Berkeley

Property Taxes and Rental Housing

Max LÖFFLER and Sebastian SIEGLOCH, Institut zur Zukunft der Arbeit, Bonn

Discussant: Owen ZIDAR 
eine größere Unabhängigkeit von der Zentralregierung honoriert worden.

Der Einfluss der eingeführten Leistungsindikatoren ist sowohl im Kontext eines theoretischen, als auch empirischen Modells analysiert worden. Da diese Indikatoren jedoch nur auf die Menge der öffentlichen Leistungen, nicht jedoch auf die Kosten abgestellt haben, ergaben sich problematische Folgen. So zeige sich, dass sowohl der Umfang der Leistungserbringung und somit der Ausgaben als auch die Steuerlast zur Finanzierung angestiegen seien. Folglich habe es keinen positiven Gesamteffekt auf die Effizienz von Kommunalpolitikern gegeben. Seine Empfehlung lautete daher, dass sinnvolle Leistungsindikatoren unbedingt auf Kosten-Nutzen-Relationen und nicht nur auf das Niveau der öffentlichen Leistungen abstellen sollten.

\section{Steuern und Umverteilung}

Christopher Balding präsentierte eine Studie zu den Ursachen der zunehmenden Volatilität der US-amerikanischen Einkommensteuereinnahmen auf Basis individueller Einkommensteuerdaten im Zeitraum von 1966 bis 2007. Die gestiegene Volatilität könne dabei auf Veränderungen in der Zusammensetzung der Einkommen zurückgeführt werden und die damit einhergehende Verschiebung der Bedeutung einzelner Einkommen für die Gesamteinnahmen. Treibender Faktor für die Volatilität in den Steuereinnahmen sei der gestiegene Anteil der Kapitaleinkommen und die Bedeutung der Spitzenverdiener für die Gesamtsteuereinnahmen im Untersuchungszeitraum.

Silvia Avram referierte zu den Verteilungseffekten von Steuerfreibeträgen und -gutschriften in sechs europäischen Ländern. Basierend auf dem Mikrosimulationsmodell Euromod, lege die empirische Analyse dar, welche Politikinstrumente eine progressive beziehungsweise regressive Wirkung entfalten würden. So zeige sich, dass im Allgemeinen Steuergutschriften progressiver seien als -freibeträ-
Who Benefits from State Corporate Tax Cuts? A Local Labor Markets Approach with Heterogeneous Firms

Owen ZIDAR, University of California, Berkeley; and Juan Carlos SUÁREZ SERRATO, Stanford University, Stanford

Discussant: Sebastian SIEGLOCH

Employment Effects of Local Corporate Taxes

Sebastian SIEGLOCH, Institut zur Zukunft der Arbeit, Bonn

Discussant: Pierre BACHAS

Not(ch) your average tax system: Corporate tax elasticities evidence from Costa Rica

Pierre BACHAS, University of California, Berkeley

Discussant: Max LÖFFLER

\section{Political Economy}

Chair: Zareh ASATRYAN, Zentrum für Europäische Wirtschaftsforschung, Mannheim

Election-induced revisions of fiscal policy outcomes: New evidence using real-time data

Jan-Egbert STURM, KOF Swiss Economic Institute, Eidgenössische Technische Hochschule Zürich; Richard JON-A-PING and Jakob DE HAAN, Rijksuniversiteit Groningen

Discussant: Robert LEHMANN

On the political economy of revenue forecasts Evidence from $O E C D$ countries

Robert LEHMANN ifo Institute Dresden; and Beate JOCHIMSEN, Hochschule für Wirtschaft und Recht, Berlin

Discussant: Marta CURTO-GRAU

Partisan targeting of inter-governmental transfers \& state interference in local elections: evidence from Spain

Marta CURTO-GRAU Rurprecht-Karls-Universität Heidelberg; Albert SOLÉ-OLLÉ and Pilar SORRIBAS-NAVARRO, Institut d'Economica de Barcelona and Universitat de Barcelona Discussant: Zareh ASATRYAN

Direct Democracy and Local Government Efficiency

Zareh ASATRYAN, Zentrum für Europäische Wirtschaftsforschung, Mannheim; and Kristof DE WITTE, Maastricht University und Katholieke Universiteit Leuven

Discussant: Jan-Egbert STURM

Public Administration II

Chair: Michael VLASSOPOULOS, University of Southampton 
ge sowie der Umstand, dass der Einfluss von Steuergutschriften als auch -freibeträgen auf die Ungleichheit relativ gering sei.

Peter Backus ging in seinem Beitrag der Frage nach, welche Determinanten die Nachfrage nach Umverteilung bestimmen. Der Fokus lag insbesondere auf der Bedeutung von Versicherungsmotiven beziehungsweise Altruismus, welcher mittels der Arbeitslosenversicherung operationalisiert wird. Die empirische Analyse nutze hierzu Veränderungen der lokalen Arbeitslosenrate, die die Nachfrage nach dem Leistungsumfang der Arbeitslosenversicherung beeinflussen würde. Die Ergebnisse zeigten, dass Beschäftigte mit einem niedrigen Risiko der Arbeitslosigkeit keine Veränderungen des Leistungsumfangs nachfragen, Beschäftigte mit einem hohen Risiko der Arbeitslosigkeit reagierten hingegen mit verstärkten Umverteilungswünschen.

Benjamin Bittschi untersuchte die Auswirkungen der deutschen Kirchensteuer auf das Spendenverhalten. Die Ergebnisse der empirischen Analyse zeigten, dass es im Allgemeinen keine ökonomisch signifikanten Crowding-out-Effekte der Kirchensteuer in Bezug auf andere Spenden gäbe. Des Weiteren würden Nicht-Spender im Vergleich zu Spendern eine höhere Austrittwahrscheinlichkeit aus der Kirche aufweisen, da NichtSpender steigende Kirchensteuerbelastungen nicht über eine Reduktion der Spenden kompensieren könnten. Schließlich zeige sich auch, dass aus der Kirche ausgetretene Personen kurzfristig ihre Spenden erhöhten, sich diese erhöhte Spendenbereitschaft allerdings nicht langfristig manifestiere.

\section{Fiskalföderalismus und Steuerwettbewerb}

Nils Soguel untersuchte für 26 Schweizer Kantone, inwiefern Regierungen von dem Mittel kreativer Buchführung Gebrauch machen, um laufende fiskalische Überschüsse zu verschleiern. Theoretische Anreize bestünden hierfür aufgrund der geltenden Fiskalregeln, welche bei Überschreitung eines bestimmten
Court's efficiency and judges' productivity

Roberto IPPOLITI, National School of Public Administration, Presidency of the Council of Ministers, Rome; Greta FALAVIGNA, CNR National Research Council - Ceris Moncalieri, Torino; and Giovanni RAMELLO, University of Eastern Piedmont, POLIS, Alessandria

Discussant: Kristof DE WITTE

Evaluating Efficient Public Good Provision: Theory and Evidence from a Generalised Conditional Efficiency Model for Public Libraries

Kristof DE WITTE, Maastricht University und Katholieke Universiteit Leuven; and Benny GEYS, BI Norwegian Business School, Oslo

Discussant: Michael VLASSOPOULOS

Are Public Sector Workers Different? Cross-European Evidence

Michael VLASSOPOULOS and Mirco TONIN, University of Southampton

Discussant: Roberto IPPOLITI

Keynote II: Efficiency and revenue administration

Michael KEEN, International Monetary Fund, Washington

\section{Local Fiscal Policy}

Chair: Ronny FREIER, Deutsches Institut für Wirtschaftsforschung, Berlin

Fiscal Effects of Municipal Amalgamation Evidence from a German State

Benedikt FRITZ, Walter Eucken Institute, Freiburg

Discussant: Ivo BISCHOFF

Policy preferences for inter-municipal cooperation: Results from a survey in three Hessian counties

Ivo BISCHOFF and Christian BERGHOLZ, Universität Kassel

Discussant: Ronny FREIER

Finding your right (or left) partner to merge. An analysis of the political determinants of municipality amalgamations

Ronny FREIER, Benjamin BRUNS, Deutsches Institut für Wirtschaftsforschung, Berlin; and Abel SCHUMANN, Organisation for Economic Co-operation and Development and Stockholm School of Economics, Stockholm

Discussant: Benedikt FRITZ 
Niveaus an Staatseinnahmen eine automatische Absenkung der Steuern erzwingen würden. Die empirische Analyse für den Zeitraum 1980 bis 2010 zeige, dass in der Tat von dem Mittel der kreativen Buchführung Gebrauch gemacht würde, um öffentliche Ausgaben zu begrenzen und Steuersätze hoch halten zu können. In Konsequenz könne daher der Rückgriff auf Mittel der kreativen Buchführung zu einer Reduktion der Staatsschulden führen.

Thushyanthan Baskaran präsentierte eine Studie zum regionalen Steuerwettbewerb zwischen deutschen Gemeinden im Kontext der Gewerbe- und Grundsteuer. Die Analyse nutze hierfür eine Reform des lokalen Finanzausgleichs in Nordrhein-Westfalen im Jahr 2003, welche zur Folge hatte, dass exogen bedingt die Gewerbe- und Grundsteuer auf Gemeindeebene deutlich erhöht wurde. Die empirische Analyse zeige dabei, dass niedersächsische Gemeinden entlang der Grenze nicht auf die geänderte Wettbewerbssituation mit angrenzenden Gemeinden in NordrheinWestfalen reagieren würden, weder kontemporär, noch graduell über einen nachfolgenden Zeitraum. Dieses Ergebnis stünde im Widerspruch zur bisherigen Steuerwettbewerbsliteratur. Diese Diskrepanz sei dadurch zu erklären, dass Letztere nicht valide Instrumente in ihren empirischen Analysen verwenden.

Eckhard Janeba präsentierte eine Studie zur Frage, ob lokale Regierungen über die Subventionierung von öffentlichen Kulturgütern im Wettbewerb um die sogenannte, creative class' stehen. Die theoretische Analyse zeige, dass die Anreize für Städte zur Subventionierung insbesondere dann sehr hoch seien, wenn es nur eingeschränkte Möglichkeiten gäbe, das Steuersystem zu diesem Zweck anzupassen. Die empirische Analyse im Kontext der Subventionierung von öffentlichen Theatern liefere Evidenz dafür, dass höhere Subventionen hochqualifizierte Bürger anziehen und die regionale Lohnspreizung verringere.

\section{Optimal Taxation}

Chair: Andreas PEICHL, Zentrum für Europäische Wirtschaftsforschung and University of Mannheim

Consuming your way to efficiency: public goods provision through non-distortionary tax lotteries Thomas GIEBE, Technische Universität Berlin; and Paul SCHWEINZER, University of York

Discussant: Vilen LIPATOV

Optimal taxation under regional inequality Vilen LIPATOV, Goethe-Universität Frankfurt; Sebastian G. KESSING and Malte ZOUBEK, Universität Siegen

Discussant: Henning BOHN

Optimal Retirement Financing: Separating Retirement from Public Pension Eligibility

Henning BOHN, University of California, Santa Barbara

Discussant: Andreas PEICHL

The world is flat: Existing tax benefit systems approximate a linear one

Andreas PEICHL, Zentrum für Europäische Wirtschaftsforschung and University of Mannheim

Discussant: Thomas GIEBE

\section{Steuerhinterziehung und Steuerplanung}

Joseph Mawejje untersuchte in seiner Studie den Zusammenhang zwischen wirtschaftlichen Rahmenbedingungen und der Steuerhinterziehung von Unternehmen in Uganda. Grundlage für die empirische Analyse bildeten Daten der ,Enterprise Surveys', welche sowohl Perzeptionsdaten zu den wirtschaftlichen Rahmenbedingungen als auch dem vermuteten Steuerhinterziehungsverhalten in der Branche erfassen. Die empirische Analyse zeige, dass insbesondere das Ausmaß der Korruption, die Effizienz im Rechtssystem und die Stabilität der Stromversorgung das Steuerhinterziehungsverhalten von Unternehmen in Uganda $\mathrm{zu}$ beeinflussen scheinen. Dieser Effekt sei jedoch bei kleinen stärker als bei großen Unternehmen ausgeprägt.

Caroline Holzmann analysierte inwiefern multinationale Unternehmen Steuerplanungs- 
möglichkeiten nutzen, die sich aus Rechtsänderungen ergeben. Analysegegenstand war das Ausmaß konzerninterner Fremdfinanzierung im Rahmen eines einmaligen Zeitfensters, welches sich zwischen der faktischen Nichtanwendbarkeit der Hinzurechnungsbesteuerung in europäischen Sachverhalten infolge eines Urteils des Europäischen Gerichtshofes aus dem Jahr 2006 und der Einführung der Zinsschranke im Jahr 2007 ergab. Auf Basis der Mikrodatenbank Direktinvestitionen der Deutschen Bundesbank zeige sich, dass das Ausmaß von Darlehen ausländischer Tochtergesellschaften an ihre deutsche Muttergesellschaft positiv mit der Länge des Gewinnverlagerungs-Zeitfensters korreliere.

Tuomas Kosonen ging der Frage nach dem Zusammenhang zwischen der Höhe des Umsatzsteuersatzes und dem Ausmaß der Umsatzsteuerhinterziehung im Kontext einer Steuerreform für Friseurdienstleistungen in Finnland nach. Im Rahmen eines Feldexperimentes sei ausgewählten Friseuren schriftlich eine erhöhte Wahrscheinlichkeit der Steuerprüfung angekündigt und im Anschluss durch die Steuerbehörden umgesetzt worden. Die Ergebnisse zeigten, dass einer höheren Prüfwahrscheinlichkeit ausgesetzte Unternehmen um bis zu fünf Prozent höhere Umsätze berichten würden im Vergleich zur Kontrollgruppe. Gleichzeitig zeige sich, dass der Umfang an angebotenen Dienstleistungen sich nicht verändere und stattdessen niedrigere Gewinnmargen sowie ein im Allgemeinen erhöhtes Hinterziehungsverhalten zu beobachten seien.

Johannes Rincke untersuchte die Wirkung extrinsischer und intrinsischer Motivation auf die Steuermoral bezüglich der Zahlung des Kirchgeldes in Bayern. Die Untersuchung greife auf Daten der kommunalen Steuerverwaltung und der Kirchenverwaltung zurück, die neben der Höhe der geleisteten Zahlung auch Informationen zum Einkommen und da- mit zur Steuerschuld enthalten, sodass eindeutig zwischen Steuerhinterziehern und ,Befolgern" unterschieden werden könne. Im Rahmen des Feldexperiments sei erstmalig eine Steuerprüfung angekündigt worden sowie die Veröffentlichung zufällig ausgewählter Steuerzahler in den Medien bei vorbildlichem Steuerverhalten. Die Ergebnisse der Studie zeigten, dass im Ausgangsfall ohne Kontrollen 20 Prozent der Steuerpflicht nachkommen. Des Weiteren sei in Folge der Ankündigung erhöhter Prüfwahrscheinlichkeiten eine zunehmende Steuermoral innerhalb der Gruppe der früheren Steuerhinterzieher festzustellen, jedoch nicht in der Gruppe der früheren ,Befolger'. Ein umgekehrter Effekt zeige sich im Falle der sozialen Belohnung, da in diesem Fall die Steuermoral ursprünglicher Steuerhinterzieher unverändert bliebe.

\section{Die Wirkung von Fiskalregeln}

Agnese Sacchi untersuchte inwieweit Fiskalregeln makroökonomische Stabilisierung in Termini von Output- und Inflationsvolatilität beeinflussen. Die empirische Analyse verwende hierzu Daten für 20 OECD-Länder ${ }^{2}$ im Zeitraum zwischen 1985 und 2012. Die Ergebnisse zeigen, dass ein großer Entscheidungsspielraum mit höherer Volatilität im Bruttoinlandsprodukt und der Inflation einhergehe. Würden jedoch strenge numerische Fiskalregeln gelten, so reduziere sich diese Volatilität. Dies helfe der Budgetpolitik einen Stabilisierungsbeitrag zu leisten. Weiterhin zeige sich, dass diese stabilisierende Wirkung eher bei Saldoregeln gegeben sei als bei Ausgaben-, Einnahmeregeln oder Schuldengrenzen.

Wildmer Daniel Gregori präsentierte eine Studie zur Wirkung von subnationalen Fiskalregeln auf öffentliche Ausgaben im Kontext italienischer Gemeinden. Die Studie betrachtete hierzu den sogenannten „Domestic Stability Pact", der von der italienischen Regierung im Jahr 1999 zur Begrenzung der

2 Organisation for Economic Co-operation and Development. 
Verschuldung von Gemeinden eingeführt worden war. Es zeige sich, dass das Design der Fiskalregeln von Relevanz für deren Effektivität sei. So bestünden Unterschiede in der Effektivität der Regeln, wenn diese öffentliche Ausgaben in ihrer Gesamtheit beschränken oder aber explizit zwischen konsumtiven und investiven Ausgaben differenzieren. Im Falle des Ersteren zeige sich, dass derart gestaltete Fiskalregeln keinen Effekt auf öffentliche Ausgaben haben. Werde zwischen Ausgabentypen differenziert, so zeige sich dieser Effekt schon. Darüber hinaus sei eine Beschränkung von konsumtiven Ausgaben durch Fiskalregeln häufig assoziiert mit einem Rückgang der durch die Gemeinden angebotenen Dienstleistungen.

Heiko Burret untersuchte am Beispiel der kantonalen Fiskalregeln in der Schweiz, inwieweit vertikale Effekte von diesen ausgehen. So bestünde die Möglichkeit im Kontext eines föderalen Staates, dass Fiskalregeln auf föderaler Ebene lediglich zu einer Verlagerung der Verschuldung auf die nächstfolgende Ebene führen könne. Die empirische Analyse konzentrierte sich auf den Zeitraum von 1980 bis 2011 und verwendete Daten zur Verschuldung, Einnahmen und Ausgaben auf kantonaler Ebene sowie für die 139 größten Städte in der Schweiz. Im Falle der Schweiz zeigte sich, dass es in dem betrachteten Zeitraum zu keiner Verschiebung der fiskalischen Last auf die nächstfolgende föderale Ebene gekommen ist.

Wolf Heinrich Reuter präsentierte eine empirische Studie zur Wirksamkeit von Fiskalregeln unter expliziter Berücksichtigung der Möglichkeit ihrer Nicht-Befolgung. Die Analyse konzentrierte sich dabei auf elf Mitgliedsländer der Europäischen Union in dem Zeitraum zwischen 1990 und 2013. Darüber hinaus erfolgte eine genaue Betrachtung der den Regeln zugrunde liegenden Gesetzestexte. Es zeige sich, dass die durch die Regeln betroffenen fiskalischen Größen dazu tendieren sich zu denen in den Regeln definierten Zielwerten zu entwickeln. Dies gelte sowohl bei einer Unter- als auch Überschreitung dieser Zielwerte. Teilweise liege dies daran, dass die in den Regeln definierten Zielwerte den langfristigen Durchschnitten der entsprechenden Fiskalvariablen entsprechen. Somit könnten Fiskalregeln auch im Falle ihrer Missachtung Einfluss auf die fiskalische Entwicklung nehmen, da sie in solch einem Fall dennoch Einfluss die Anpassungsgeschwindigkeit nehmen würden.

\section{Die Inzidenz der Besteuerung}

Max Löffler untersuchte in seinem Beitrag die Inzidenz der Grundsteuer in Deutschland. Dies sei insbesondere deshalb von Bedeutung, da Mieten und Hypotheken einen wesentlichen Teil des Budgets der Haushalte ausmachten. Die Ergebnisse zeigten dabei, dass es kurzfristig die Haus- und Grundstücksbesitzer sind, die die Steuerlast tragen würden. Langfristig zeige sich hingegen, dass eine Abwälzung der Steuerlast auf die Mieter stattfinde. Dies hänge von verschiedenen Immobilieneigenschaften $a b$. Im Falle neuerer Immobilien und jenen hoher Qualität, verbleibe die Steuerlast hauptsächlich beim Besitzer.

Owen Zidar ging der Frage nach, welche Auswirkungen die Gewerbesteuer auf das Entscheidungsverhalten zur Ansiedlung von Arbeitnehmern, Unternehmens- und Grundstücksbesitzern hat. Die theoretische und empirische Analyse unterstelle hierzu, dass nicht nur Unternehmen, sondern auch Arbeitnehmer mobil sind und somit auf den Steuersatz reagieren. Unterschiede in der Gewerbesteuer auf der Ebene der US-Bundesstaaten werden genutzt, um die Wirkung von Steuersatzänderungen auf die Ansiedlungsentscheidung von Unternehmen, Arbeitnehmern und die Höhe von Gehältern und Mietpreisen zu untersuchen. Im Gegensatz zu bisherigen Ergebnissen, die mobile Unternehmen und immobile Arbeitnehmer unterstellen würden, zeige sich, dass Firmen über einen Zeittraum von zehn Jahren nur doppelt so mobil wie Arbeitskräfte seien. Die Steuerlast würde dabei 
$\mathrm{zu} 40$ Prozent von Unternehmenseigentümern, zu 35 Prozent von Arbeitnehmern und zu 25 Prozent von Grundstückseigentürmern getragen.

In einer inhaltlich verwandten Studie untersuchte Sebastian Siegloch die Beschäftigungswirkung der Körperschaftssteuer in Deutschland. Hierbei nutze die empirische Analyse Unterschiede in den Hebesätzen auf Gemeindeebene zur Analyse der Beschäftigungswirkung auf ansässige Unternehmen. Die Studie zeige, dass die Beschäftigung nach einer Erhöhung des Hebesatzes reduziert würde, jedoch nach einem Zeitraum von drei Jahren wieder ein vergleichbares Niveau wie vor der Steuererhöhung erreiche. Des Weiteren zeige sich, dass eine durchschnittliche Erhöhung der Steuerlast für Unternehmen in Höhe von einem Euro die Lohnsumme um durchschnittlich 20 Cent reduziere.

Pierre Bachas analysierte Verhaltensreaktionen von Unternehmen in Costa Rica entlang von Sprungstellen im Unternehmensteuertarif. Theoretische Modelle vermuteten in diesem Zusammenhang, dass eine große Zahl an Unternehmen vor der Sprungstelle zu finden sei, während sich hinter dieser nur wenige befänden, da bei ihrer Überschreitung die Steuerlast überproportional steige. Die empirische Untersuchung zeige, dass eine Konzentration von Unternehmen an den Sprungstellen beobachtet werden könne, jedoch keine Lücke nach dieser zu finden sei. Des Weiteren zeige sich, dass insbesondere profitablere Firmen dazu neigen würden sich vor den Sprungstellen anzusiedeln.

\section{Politische Ökonomie und Föderalismus}

Jan-Egbert Sturm untersuchte das Prognoseverhalten von Regierungen für Fiskaldaten. Die Analyse betrachte halbjährlich veröffentlichte Prognosen im Kontext des „OECD Economic Outlook" für 25 OECD-Staaten im Zeitraum zwischen 1997 und 2006. So zeige sich, dass Regierungen immer wieder erhebliche Korrekturen in ihren Prognosen vorneh- men müssen. Darüber hinaus deute die empirische Evidenz darauf, dass diese Korrekturen nicht zufällig oder technisch bedingt seien, sondern systematisch geschehen würden und eine politische Motivation hätten. So würden in Jahren vor Wahlen Prognosen zu Staatsausgaben systematisch nach unten korrigiert und ebenso Daten zu Staatseinnahmen. Die empirische Evidenz deute daraufhin, dass amtierende Regierungen in Jahren vor Wahlen hierdurch einen kleinen Staat und somit Kompetenz signalisieren wollten.

Robert Lehmann präsentierte eine verwandte Studie. Hier lag der Fokus jedoch auf den Prognosen zu Steuereinnahmen. Erneut stammte die Datengrundlage aus 18 OECDStaaten für den Zeitraum von 1996 bis 2012. Die Ergebnisse deuteten darauf hin, dass linke Regierungen stärker zur Überschätzung der Steuereinnahmen neigen als rechte Regierungen. Darüber hinaus deute die empirische Evidenz darauf hin, dass eine zunehmende politische Fragmentierung zu pessimistischeren Prognosen im Bereich der Steuereinnahmen führe.

Marta Curto-Grau untersuchte den Einfluss politischer Ideologie auf die Allokation vertikaler staatlicher Transfers im spanischen Föderalismus. Der Fokus lag dabei auf den durch die regionalen Regierungen an Gemeinden weiterverteilten Staatseinnahmen. So zeige sich, dass regionale Regierungen polit-ideologisch gleichgesinnte Gemeinden bei der Allokation von Transfers bevorzugen würden. Dieser Effekt verstärke sich weiter, wenn Wahlen auf der regionalen Ebene weniger kompetitiv sein.

Zareh Asatryan präsentierte eine Studie zu dem Einfluss direkter Demokratie auf die Effizienz kommunaler Regierungen. Datengrundlage bildeten über 2.000 bayerische Gemeinden und die über 2.500 direktdemokratischen Initiativen. Die Effizienz sei anhand sechs verschiedener Ausgabenkategorien beziehungsweise Dienstleistungen gemessen worden. Die empirische Evidenz deute da- 
rauf, dass mehr direkte Demokratie mit effizienteren kommunalen Regierungen assoziiert zu sein scheint.

\section{Die Effizienz der öffentlichen Verwaltung}

Roberto Ippoliti präsentierte eine Studie zur Effizienz der Steuergerichtsbarkeit in Italien. Der Fokus lag dabei insbesondere auf der Rolle der vorherrschenden Anreizwirkungen für Richter. Die Steuergerichtsbarkeit in Italien weise in diesem Zusammenhang die Besonderheit auf, dass lediglich der Präsident des Gerichts hauptberuflich in dieser Kammer tätig und ein Steuerrechtsexperte sein müsse. Die weiteren am Gericht tätigen Richter müssten nicht ausschließlich in dieser Kammer beschäftigt sein und haben auch die Möglichkeit, in der Privatwirtschaft zu arbeiten. Die Anreize für die hauptamtliche Beschäftigung am Gericht seien jedoch niedrig, da Richter aus dieser Gruppe lediglich durch eine niedrige Fallpauschale vergütet würden. Die empirische Evidenz zeige daher, dass die Absenkung der Opportunitätskosten für Richter die zentrale Größe zur Steigerung der Effizienz der Steuergerichtsbarkeit sei.

Kristof De Witte untersuchte die effiziente Bereitstellung öffentlicher Güter an dem Beispiel öffentlicher Bibliotheken in Belgien. Zentrale methodische Innovation bei der Effizienzanalyse sei die Berücksichtigung der Tatsache, dass öffentliche Güter stets in Koproduktion bereitgestellt würden. Dieser Umstand führe dazu, dass klassische Outputmaße nicht geeignet seien, die Effizienz der öffentlichen Verwaltung zu messen, und es daher notwendig sei, stattdessen das Servicepotenzial zu messen. Vor diesem Hintergrund zeigten die Ergebnisse, dass mit steigendem Wohlstand, mit zunehmender Bevölkerungsdichte und im Falle von links gerichteten regionalen Regierungen die Effizienz der öffentlichen Verwaltung zunehme.

Michael Vlassopoulos präsentierte eine Studie zu den Determinanten für die sogenannte
„Public Sector Motivation“3 von Mitarbeitern im öffentlichen Sektor. Frühere Studien hätten Evidenz für eine systematisch höhere „Public Sector Motivation“ unter Mitarbeitern des öffentlichen Sektors im Vergleich zum privaten Sektor gefunden. Diese Studien basierten jedoch häufig auf Umfragedaten unter Berufstätigen und berücksichtigten nicht die Heterogenität in Jobcharakteristika zwischen beiden Sektoren. Unter Verwendung von Umfragedaten unter Rentnern aus beiden Sektoren und unter Berücksichtigung struktureller Unterschiede zwischen beiden Sektoren zeige sich, dass ein stärker ausgeprägtes Sozialverhalten eher in Führsorgeberufen festzustellen sei, jedoch nicht im Allgemeinen für den öffentlichen Sektor zu konstatieren sei.

\section{Der Einfluss der Struktur der Steuerverwal- tung}

Michael Keen befasste sich in seinem Vortrag mit Ansätzen zur Steigerung der Effizienz in der Steuerverwaltung. Der Fokus lag dabei sowohl auf organisatorischen Ansätzen als auch auf Leistungsindikatoren. Im Falle der Entwicklungshilfe durch den Internationalen Währungsfonds sei ein Trend in Entwicklungsländern, der Finanzverwaltung eine größere Eigenständigkeit gegenüber der allgemeinen öffentlichen Verwaltung zu geben. Obwohl theoretisch ein positiver Effekt zu erwarten wäre, bliebe die Wirkung der gestiegenen politischen Eigenverantwortung in Bezug auf die Korruptionsbekämpfung jedoch unklar. Entsprechendes gelte auch für eine alternative organisatorische Ausgestaltung der Finanzverwaltung entlang von Steuertypen.

Keen stellte auch alternative Ansätze unter Verwendung von Leistungsindikatoren vor. So seien Erhebungskosten als Indikatoren zwar nicht geeignet, neue Indikatoren zur Messung der Ausschöpfung des Steuerpotenzials seien hingegen geeignet. Die auf diese Weise bestimmten ,Steuer-Lücken' könnten daraufhin zerlegt werden in Lücken bedingt

3 Die bewusste Entscheidung beziehungsweise intrinsische Motivation im öffentlichen Sektor tätig zu sein. 
durch defizitäre Gesetzgebung sowie in Lücken bedingt durch Defizite im Steuervollzug. Somit könne dieser Typ von Leistungsindikatoren sehr informativ sein, Defizite in der Steuerdurchsetzung aufzudecken.

\section{Wirkung und Determinanten von Gemeinde- zusammenlegungen und-kooperationen}

Benedikt Fritz präsentierte eine Studie zu den Wirkungen von Gemeindezusammenlegungen auf fiskalische Größen. Untersuchungsgegenstand waren dabei Zusammenlegungen im Kontext der Gemeindegebietsreform in Baden-Württemberg zwischen 1967 und 1974. Die Ergebnisse der empirischen Analyse deuten darauf hin, dass zusammengelegte Gemeinden sich in darauffolgenden Jahren stärker verschulden würden und dieser Anstieg hauptsächlich durch zunehmende Ausgaben für Verwaltungspersonal und öffentliche Bauprojekte determiniert sei.

Ivo Bischoff befasste sich in seinem Vortrag mit einer verwandten Fragestellung zu Gemeindekooperationen in Hessen. Insbesondere Gemeinden in der Peripherie von Metropolregionen seien häufig keine attraktiven Partner für Gemeindezusammenlegungen, sodass sich hier vielfach nur das Mittel der Gemeindekooperation anböte. Die Studie untersuchte daher die Präferenzen der Bevölkerung hinsichtlich dieser Mittel mithilfe von Umfragedaten für 60 hessische Gemeinden. So zeige sich, dass es keinen grundsätzlichen Widerstand gegen dieses Mittel der Zusammenarbeit gäbe und insbesondere technische Aufgaben im Kontext der Gemeindekooperation akzeptiert seien. Darüber hinaus gäbe es eine größere Zustimmung für Kooperationen mit Gemeinden vergleichbarer Größe.

Ronny Freier untersuchte den Einfluss der Parteizusammensetzung der Gemeinderäte auf die Wahrscheinlichkeit für Gemeindezusammenlegungen. Untersuchungsgegenstand waren in diesem Fall Gemeinden in Brandenburg und politische Reformen im Zeitraum von 1999 bis 2003. Die Studie nutzt dabei eine Besonderheit des Reformprozesses, welcher zunächst freiwillige Gemeindezusammenlegungen vorsah und diese erst im Anschluss für verpflichtend erklärte. So zeigen die empirischen Ergebnisse, dass die politideologischen Ausrichtung der Gemeinderäte einen signifikanten Effekt auf die Wahrscheinlichkeit für eine Gemeindezusammenlegung in der freiwilligen Phase habe.

\section{Optimale Besteuerung}

Thomas Giebe ging im Rahmen einer theoretischen Analyse der Frage nach der optimalen Steuerfinanzierung öffentlicher Güter nach. Der Fokus lag insbesondere auf der kritischen Analyse bisheriger Forschungsergebnisse, die Ineffizienzen in der Finanzierung öffentlicher Güter durch die Besteuerung privaten Konsums erklärten. Die Ergebnisse der theoretischen Analyse zeigten, dass es möglich sei, mithilfe von sogenannten ,Steuerlotterien“ Verzerrungen im privaten Konsum zu vermeiden, öffentliche Güter im sozial optimalen Umfang bereitzustellen sowie den Staatshaushalt hierdurch nicht zusätzlich zu belasten.

Vilen Lipatov analysierte die Wirkung regionaler Produktivitätsunterschiede auf die optimale Ausgestaltung von Steuer-Transfer-Systemen. Zentrales Merkmal der theoretischen Analyse sei die Berücksichtigung der Möglichkeit produktivitätssteigernder Migration von Arbeitskräften in solch einem Kontext. Die Ergebnisse der Studie zeigten, dass regionale Produktivitätsunterschiede eine zusätzliche Restriktion für die Ausgestaltung von Steuer-Transfer-Systemen darstellen würden und die Umverteilungswirkung einschränken könnten. Des Weiteren führe die Möglichkeit der produktivitätsbedingten Migration von Arbeitskräften tendenziell zu einer Reduktion der Grenzsteuersätze.

Henning Bohn untersuchte im Rahmen einer theoretischen Analyse ein neues Konzept für die Ausgestaltung eines Zwei-Säulen-Rentensystems. Zentrales Element hierbei sei eine 
veränderte Konzeptualisierung der Komplementarität von privater und staatlicher Vorsorge. Demnach solle sich die private Vorsorge auf die Absicherung der Anfangsjahre des Ruhestands und die staatlich organisierte Alterssicherung auf die letzten Lebensjahre konzentrieren. Die theoretische Analyse zeige dabei, dass solch ein Konzept einen komparativen Vorteil in Modellkontexten mit langen Zeithorizonten aufweise.

Andreas Peichl untersuchte auf Basis von Daten für 26 europäische Nationen und die USA die De-facto-Ausgestaltung der vorherr- schenden Steuer-Transfer-Systeme. Die Analyse berücksichtigt dabei sowohl direkte Steuern auf Seiten der Haushalte als auch Unternehmenssteuern, indirekte Steuern sowie Sachleistungen. Die Ergebnisse der empirischen Analyse zeigten, dass unter Berücksichtigung des gesamten Steuer-Transfer-Systems das Verhältnis zwischen Nettosteuerzahlungen und dem Bruttoeinkommen einem linearen Zusammenhang und somit einem Flat-Tax-System gleichkomme. So könne ein lineares Steuersystem bis zu 94 Prozent der Variation in den existieren Steuersystemen erklären.

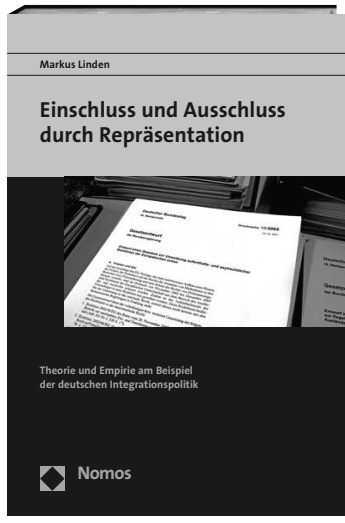

Einschluss und Ausschluss durch Repräsentation Theorie und Empirie am Beispiel der deutschen Integrationspolitik Von Markus Linden 2014, 437 S., brosch., 39,-€ ISBN 978-3-8487-1789-7 www.nomos-shop.de/23514

Wann ist politische Repräsentation möglichst inklusiv? Der Autor skizziert die Diskussion, entwirft eine eigene Repräsentationstheorie und überprüft diese anhand der Repräsentation von Migranteninteressen in Deutschland seit 1998. Gängige Urteile der öffentlichen Debatte werden dabei relativiert.

Bestellen Sie jetzt telefonisch unter 07221/2104-37 Portofreie Buch-Bestellungen unter www.nomos-shop.de Alle Preise inkl. Mehrwertsteuer
Nomos 